\title{
Relación con los padres y estrategias de afrontamiento en adolescentes de Lima
}

\author{
Relationship with parents and coping strategies in adolescents of \\ Lima
}

\section{Tomás P. Caycho}

Universidad de San Martín de Porres, Lima, Perú.

Doctorando en Psicología. Docente de Métodos de Investigación y Psicología experimental e Investigador Interno del Instituto de Investigación.

Recibido: $18-01-16$

Aprobado: 19-02-16

\section{Correspondencia}

Email: tcaycho@usmp.edu.pe

\section{Citar como:}

Caycho, T. (2016). Relación con los padres y estrategias de afrontamiento en adolescentes de Lima. Propósitos y Representaciones, 4(1), 11-59. doi: http://dx.doi.org/10.20511/ pyr2016.v4n1.86

\footnotetext{
(C) Universidad San Ignacio de Loyola, Vicerrectorado de Investigación y Desarrollo, 2016.

(c) BY-NC-ND Este artículo se distribuye bajo licencia CC BY-NC-ND 4.0 Internacional (http://creativecommons.org/licenses/by-nc-nd/4.0/).
} 


\section{Resumen}

El presente estudio, de diseño correlacional y comparativo, tiene por objetivo determinar la relación entre la percepción de la relación con los padres y las estrategias de afrontamiento en una muestra de 320 adolescentes de Lima, elegidos a través de un muestreo no probabilístico, 156 hombres $(48.75 \%)$ y 164 mujeres (51.25\%). Se emplearon, como instrumentos de recojo de información, la Children's Report of Parental Behavior Inventory y la Escala de Afrontamiento para Adolescentes. Los resultados permiten concluir que existen correlaciones estadísticamente significativas entre algunas dimensiones de la percepción de la relación con los padres y las estrategias de afrontamiento en la muestra estudiada. Finalmente, respecto a la percepción de los estilos parentales de la madre y el padre, podemos observar que no se evidencian diferencias significativas entre hombres y mujeres, con excepción de la autonomía extrema del padre, en la cual los varones puntúan más alto que las mujeres. Al analizar el afrontamiento en la muestra en relación con el sexo, no se encontraron algunas diferencias estadísticamente importantes.

Palabras clave: Adolescentes, estrategias de afrontamiento, relación con los padres.

\section{Summary}

This correlational and comparative study aims to determine the relationship between the perception of the relationship with parents and coping strategies in a sample of 320 students chosen through a non-probabilistic sampling of 156 men (48.75\%) and 164 women (51.25\%). To that end, information gathering instruments like the Children's Report of Parental Behavior Inventory and Adolescent Coping Scale were used. The results suggest that there are statistically significant correlations between some dimensions of perception of the relationship with parents and coping strategies in the sample studied. Finally, with regard to the perception of parenting styles of 
both mother and father, we see no significant differences between men and women, except for the extreme autonomy of the father, in which men score higher than women. There were no some statistically significant differences in the analysis of coping strategies in the sample in relation to gender.

Keywords: Adolescents, coping, relationship with parents 


\section{Introducción}

La adolescencia es una etapa caracterizada por el enfrentamiento a nuevas situaciones, como las modificaciones corporales, la elección de pareja o profesión, nuevas formas de pensamiento, la definición de la propia identidad, el establecimiento de cierta independencia emocional de los padres, etc., las cuales pueden resultar estresantes. Ante esto, es necesario contar con recursos que posibiliten a los adolescentes un manejo exitoso de este tipo de eventos, así como de capacidades para un empleo eficaz, razonable y flexible de los mencionados recursos. Entre los factores personales que facilitan el desarrollo de conductas adaptadas destacan las relaciones familiares y las estrategias de afrontamiento.

Con respecto a la percepción de las relaciones con los padres, estas pueden ser definidas como una combinación de actitudes hacia los hijos que generan un clima emocional en el cual los padres actúan (Darling \& Steinberg, 1993). Así, la percepción de las relaciones con los padres ayuda en la comprensión de las conductas y actitudes de ellos, y cómo estas se relacionan con el desarrollo y bienestar de los hijos (Domenech, Donovick \& Crowley, 2009). La percepción de los estilos parentales se basa en variaciones de las dimensiones parentales de sensibilidad (calidez, afecto), exigencia paterna (control parental) y autonomía brindada. La evidencia señala que las dimensiones parentales mencionadas se relacionan con el desarrollo y bienestar de los hijos (Broderick \& Blewitt, 2003).

Las dimensiones parentales de sensibilidad, control parental y autonomía brindada coinciden con otras clasificaciones como la propuesta por Baumrind (1966) y Maccoby y Martin (1983), referidas a la aceptación (parental responsiveness), control patológico (parental demandingness) y negligencia (uninvolved or neglectful parenting); así como la de Schaefer (1965), que propone un modelo tridimensional constituido por tres pares de factores ortogonales denominados: 1) Aceptación frente al rechazo; 2) 
Autonomía psicológica frente al control psicológico (control parental a través de la dominación, generando culpa y ansiedad); 3) Control firme frente al control laxo (control parental a través de la imposición de reglas y establecimiento de límites).

A partir de un modelo cognitivo, se menciona que lo que influye en mayor medida en el comportamiento de los adolescentes es la percepción que estos tienen del tipo de relación con sus padres más que el comportamiento de los mismos (Richaud de Minzi, 2005). Es así que la percepción, por parte de los hijos, de que sus padres les brindan apoyo se asocia con un aumento de su autoestima, su integración social, la percepción de control y la efectividad de sus afrontamientos, y cómo estas ayudan a la comprensión de las conductas y actitudes de los padres, y cómo se relacionan con el desarrollo y bienestar de los hijos (Domenech, Donovick \& Crowley, 2009; Sandler, Wolchick, MacKinnon, Ayers \& Roosa, 1997). Así mismo, la calidad de las relaciones familiares percibidas por los hijos se asocia con la habilidad de los adolescentes para afrontar los problemas (Sandler, et al., 1997; Skinner \& Welborn, 1994).

Efectivamente, durante la adolescencia, tanto varones como mujeres hacen frente a diversos estresores derivados de la aparición de conflictos maduracionales, de grupo de pares, diferencias familiares, de identidad, entre otros (Richaud de Minzi, 2003). Estudios con adolescentes señalan los efectos negativos del estrés sobre la atención, concentración, conductas adaptativas (Caballero, Abelló \& Palacios, 2007), procesos de aprendizaje (Martín, 2007), bienestar psicológico (Guarino, Gavidia, Antor \& Caballero, 2000) y salud en general (Sarid, Anson, Yaari \& Margalith, 2004), entre otros. El género también tiene incidencia en la experiencia del estrés, siendo las mujeres quienes presentan mayores niveles de estrés percibido en comparación con los hombres (Calais, Andrade \& Lipp, 2003; Heiman, 2004). 
La teoría transaccional del estrés enfatiza la evaluación y percepción sobre las posibles consecuencias representadas por experiencias ambientales objetivas (Cohen, Kessler \& Underwood, 1995). A partir de este enfoque se describen dos procesos que tienen lugar en la relación individuoentorno: la evaluación cognitiva y el afrontamiento. Siguiendo a Lazarus y Folkman (1986), podemos encontrar dos tipos de estilo de afrontamiento: el primero, enfocado en el problema, y el segundo, centrado en la emoción. El afrontamiento centrado en el problema incluye el uso de estrategias de solución de problemas, toma de decisiones, búsqueda de información, etc., las cuales son empleadas cuando la persona tiene por objetivo realizar un cambio de la situación estresante. El afrontamiento centrado en la emoción se enfoca en la modificación de la reacción emocional, a través del empleo de la técnica de reestructuración cognitiva. Estas estrategias de afrontamiento, también llamadas estrategias cognitivas de afrontamiento, se presentan en casos en que las personas consideran que es difícil realizar algo para modificar la situación estresante, no quedando más alternativa que resistirlo (Lazarus \& Folkman, 1986).

La literatura señala que las personas emplean una amplia variedad de estrategias para manejar diferentes situaciones estresantes. El uso de una estrategia, así como el éxito de la misma, dependerá de la naturaleza del evento estresor (Taylor, 2007).

Frydenberg (1993), en base a los postulados de Lazarus, conceptualiza el afrontamiento adolescente como la expresión de una serie de acciones en respuesta a demandas planteadas a la persona. Estas acciones o conductas pueden tener diferentes objetivos: primero, pueden estar dirigidas a modificar la fuente del estrés; otras permiten adaptarse a la demanda; y, finalmente, algunas acciones indican una incapacidad para manejar las diferentes demandas. El modelo enfatiza también la revaluación del resultado (Frydenberg, 1997; Frydenberg y Lewis, 1997). Así, cada individuo, luego de una valoración de la situación, evalúa el impacto del 
estrés, por lo cual concluye si las consecuencias llevan al daño, amenaza o desafío, además de estimar los recursos con los que cuenta. De acuerdo con esto, la nueva respuesta es revaluada, pudiendo originarse una nueva respuesta. Se origina, de esta manera, un mecanismo de retroalimentación que brinda información para tomar la decision de si las estrategias empleadas serán nuevamente utilizadas o serán descartadas, según el resultado de su evaluación.

Diversos estudios que buscan relacionar las estrategias de afrontamiento y la percepción de la relación con los padres señalan que la calidez, la intimidad y la comunicación con los hijos brindada por los padres predicen una mayor utilización de estrategias de acción sobre el problema (Herman \& McHale, 1993). Así mismo, quienes percibían a sus padres como indulgentes y negligentes utilizan un afrontamiento más cognitivo, mientras que percibir a los padres como más cálidos y como fuente de apoyo se relaciona con un afrontamiento centrado en el problema. Finalmente, la percepción de los padres como de mayor firmeza y vigilancia se relaciona con un afrontamiento centrado en la emoción y cognitivo (Dusek \& Danko, 1994). La calidez parental percibida se asocia positivamente con el afrontamiento activo; mientras que quienes perciben un estilo de crianza autoritario y permisivo por ambos padres muestran un afronte activo a los problemas (Wolfradta, Hempelb \& Miles, 2003).

Otros estudios (Richaud de Minzi, 2006) indican que la percepción de las relaciones con los padres influye en el afrontamiento de los adolescentes, de acuerdo con el sexo y la edad. Así, el sentirse amado y valorado por los padres favorece el desarrollo de estrategias de afrontamiento funcionales; mientras que el control patológico se relaciona con estrategias no adaptativas o disfuncionales cuando se enfrenta a los conflictos, especialmente la dependencia emocional, auto-culpa y parálisis. Por último, la percepción de la negligencia en ambos padres, básicamente, conduce a la solución de la evasión y la falta de responsabilidad. Richaud de Minzi, Moreno y Sacchi 
(2011) señalan que cuando el adolescente se siente aceptado desarrolla un afrontamiento funcional basado en la reestructuración cognitiva, la búsqueda de apoyo emocional y la acción sobre el problema. Al mismo tiempo, parecería que el percibir aceptación de parte de sus padres lo defiende de desarrollar un afrontamiento disfuncional basado en la evitación cognitiva, el descontrol y la paralización. En cambio, cuando percibe en sus padres rechazo, hostilidad, culpabilización y restricción de su autonomía, desarrolla un afrontamiento disfuncional frente al conflicto. Si bien el control aceptado es considerado muy importante en un estilo de relación positivo, el control excesivo, que coarta la autonomía y la seguridad del adolescente, es altamente negativo para un afrontamiento exitoso de la amenaza.

Con el fin de poder estudiar la asociación entre la percepción de las relaciones con los padres y los estilos de afrontamiento, se ha elegido investigarla en adolescentes universitarios de Lima Metropolitana, ya que son variables importantes para explicar el ajuste de dichos adolescentes a la situación académica que experimentan y permiten establecer un pronóstico de adaptación a su vida académica futura.

La importancia del presente estudio podemos encontrarla a nivel teórico y práctico. En relación con el primero, los resultados obtenidos contribuyen brindando conocimientos científicos acerca de dos constructos psicológicos importantes: la percepción de las relaciones con los padres y el afrontamiento, considerados relevantes para explicar parte de la adaptación frente a las diversas demandas del medio (Herman \& McHale, 1993). Así mismo, los resultados buscan aportar dentro del area de la psicología del desarrollo en el contexto peruano, ya que brindan un perfil de la manera en que los adolescentes enfrentan diversas situaciones de estrés a través del empleo de diferentes estrategias. En el sentido aplicado, los resultados pueden brindar información útil para el diseño de programas de prevención 
primaria y secundaria en población adolescente que estén más acorde con sus necesidades. Frente a un sistema universitario altamente competitivo, los adolescentes requieren de habilidades de afronte ante este y otros problemas a lo largo de su vida. Así, el conocimiento de estas variables permitiría un mejor conocimiento de los mecanismos más favorables para sortear periodos de estrés de una mejor manera.

\section{Método}

La presente investigación tiene un enfoque cuantitativo. Se trata de un estudio de tipo no experimental con un diseño transeccional correlacional ex post facto, ya que tiene como propósito establecer y analizar la relación entre dos o más variables; además, se busca conocer si la percepción de la relación con los padres se vincula con el empleo de las estrategias de afrontamiento de un grupo de adolescentes universitarios de Lima (Hernández, Fernández, \& Baptista, 2006).

\section{Participantes.}

Se trabajó con 320 estudiantes, elegidos a través de un muestreo no probabilístico: 156 hombres (48.75\%) y 164 mujeres (51.25\%), que se encuentran estudiando en universidades públicas y privadas de Lima Metropolitana. La Tabla 1 presenta las características de la muestra, en relación con la distribución de edades, lugar de nacimiento y facultad en la que estudia. 


\section{Tabla 1.}

Características sociodemográficas de la muestra.

\begin{tabular}{lcc}
\hline & Frecuencia & Porcentaje \\
\hline Edad & 110 & \\
16 & 90 & $34.375 \%$ \\
17 & 120 & $28.125 \%$ \\
18 & & $37.5 \%$ \\
Lugar de nacimiento & 260 & \\
Lima & & $81.25 \%$ \\
Provincia & 60 & $18.75 \%$ \\
Facultad & & \\
Psicología & 220 & $68,7 \%$ \\
Trabajo Social & 100 & $31.25 \%$ \\
\hline
\end{tabular}

\section{Instrumentos.}

Para los propósitos de la investigación se emplearon dos instrumentos: la Versión Abreviada del Inventario de la Percepción de los Hijos acerca de las Relaciones con sus Padres y la Escala de Afrontamiento para Adolescentes. El Children's Report of Parental Behavior Inventory (CRPBI), creado por Schaefer (1965), es un instrumento a través del cual los hijos describen la percepción que tienen del comportamiento de los padres hacia ellos. Está basado en un modelo tridimensional resultante del análisis factorial, que permitió identificar tres pares de factores ortogonales: Aceptación versus Rechazo, Autonomía Psicológica vs. Control Psicológico (control parental a través de la dominación y la instilación de culpa y ansiedad) y Control Firme vs. Control Laxo (control parental a través de la imposición de reglas y establecimiento de límites). La versión original consta de 26 escalas de 10 ítems cada una, con un formato de respuesta dicotómico (Sí, No).

Richaud de Minzi (2005) elaboró una versión de 32 ítems que, a su vez, fueron re-factorizados. Tanto la forma para la madre como para el padre da información acerca de tres factores: Aceptación, Control Patológico 
y Autonomía Extrema, que apuntan a modos de relación generales, dentro de los cuales se han incluido, de manera equilibrada, formas más específicas de interacción propuestas por Schaefer. Esta nueva versión abreviada ha demostrado una adecuada validez de criterio, al ser utilizada para estudiar la relación entre los estilos parentales percibidos y el afrontamiento en los adolescentes, obteniéndose resultados en la dirección esperada según la teoría. Al mismo tiempo, a pesar de la reducción en el número de ítems, la fiabilidad como consistencia interna ha sido satisfactoria. Así, se calculó el coeficiente de Cronbach para cada uno de los tres factores, obteniéndose índices de fiabilidad de 0.81 en aceptación, 0.83 en control patológico y 0.75 en autonomía extrema, con respecto a la madre, y de 0.82 en aceptación, 0.84 en control patológico y 0.73 en autonomía extrema, con respecto al padre.

La Escala de Afrontamiento para Adolescentes, de Frydenberg \& Lewis (1997), evalúa cómo el adolescente entre 12 y 18 años afronta sus problemas en general. Concretamente, se diferencian 18 estrategias de afrontamiento que pueden agruparse en tres estilos básicos de afrontamiento: 1) Dirigido a la resolución del problema, reúne una serie de estrategias tendientes a modificar la situación problemática para hacerla menos estresante, tales como: buscar diversiones relajantes, distracción física, esforzarse y tener éxito, fijarse en lo positivo y concentrarse en resolver el problema; 2) Afrontamiento en relación con los demás, reúne estrategias como: buscar apoyo espiritual, invertir en amigos íntimos, buscar ayuda profesional, buscar apoyo social, buscar pertenencia y acción social; 3) Afrontamiento improductivo, concentra estrategias que tienen que ver con: autoinculparse, hacerse ilusiones, ignorar el problema, falta de afrontamiento, preocuparse, reservarlo para sí y reducción de la tensión.

La versión original argentina arroja, mediante un análisis de la consistencia interna, un alfa de Cronbach de 0.75 . En el contexto peruano, Caycho, Castilla y Shimabukuro (2014) obtuvieron evidencias de validez y confiabilidad que apoyan su uso en muestras de adolescentes El análisis 
de correlación ítem-test encontró asociaciones altamente significativas $(p<$ .001) para cada uno de los reactivos. Así mismo, se obtiene una elevada consistencia interna ( $\alpha$ de Cronbach $=0.88)$; mientras que el análisis factorial realizado reveló que la ACS presenta una estructura de once factores.

\section{Procedimiento.}

Las pruebas fueron administradas en forma colectiva. Antes de que los estudiantes dieran respuesta a los ítems, se les pidió que leyeran cuidadosamente las instrucciones de cada prueba, recordándoles que no existe límite de tiempo para responder a ambas escalas. Los participantes completaron los datos referentes a edad, género, estado civil y situación laboral, conservando su anonimato en diligenciamiento de las escalas. Los estudiantes participaron de forma voluntaria y anónima, firmando el consentimiento informado que garantiza la confiabilidad de los datos suministrados. En relación con el análisis de datos, se utilizó la versión 22 del Statistical Package for the Social Sciences (SPSS).

\section{Resultados}

\section{Análisis correlacionales.}

En primer lugar, se observan las correlaciones y el nivel de significación entre los tres estilos parentales del padre, percibidos por los hijos, y las estrategias de afrontamiento (Tabla 2). Se observa que existen correlaciones negativas y estadísticamente significativas entre reestructuración cognitiva y control patológico $(r=-0.444, p<.05)$, ansiedad y autonomía extrema $(r=$ $-0.390, p<.05)$, y control patológico y actividades de aislamiento $(r=-0.377$, $p<.05)$. 


\section{Tabla 2.}

Correlaciones entre los tres estilos de la relación con el padre y las once estrategias de afrontamiento.

\begin{tabular}{lccc}
\hline & $\begin{array}{c}\text { Control } \\
\text { patológico }\end{array}$ & Aceptación & $\begin{array}{c}\text { Autonomía } \\
\text { extrema }\end{array}$ \\
\hline Reestructuración cognitiva & $\mathbf{- 0 . 4 4 4 ^ { * }}$ & -0.114 & -0.186 \\
Autoinculparse & -.095 & 0.250 & -0.186 \\
Fatalismo & -0.195 & -.078 & -.087 \\
Evasión a través de la diversión & -.014 & -.044 & -0.101 \\
Focalizado en el problema & 0.241 & .047 & -0.197 \\
Evasión a través de la actividad física & 0.192 & -0.290 & .070 \\
Apoyo emocional & .036 & -.061 & -0.125 \\
Descarga emocional y somatización & -0.190 & 0.144 & .070 \\
Ansiedad & .084 & -.094 & $-\mathbf{0 . 3 9 0}$ \\
Aislamiento & $-\mathbf{0 . 3 7 7 ^ { * }}$ & .031 & 0.107 \\
No acción & -0.365 & -.026 & -.052 \\
\hline
\end{tabular}

$* \mathrm{p}<.05$

La Tabla 3 permite observar las correlaciones y el nivel de significación entre los tres estilos de relación con la madre percibidos por los hijos y las estrategias de afrontamiento. Se observa que el autoinculparse presenta correlaciones estadísticamente significativa y negativa con el control patológico $(r=-0.620, p<.01)$ y positiva con la aceptación $(r=$ $0.566, p<.01)$. Finalmente, encontramos una correlación negativa entre el control patológico y actividades de aislamiento $(r=-0.398, p<.01)$. 


\section{Tabla 3.}

Correlaciones entre los tres estilos de relación con la madre y las once estrategias de afrontamiento.

\begin{tabular}{lccc}
\hline & $\begin{array}{c}\text { Control } \\
\text { patológico }\end{array}$ & Aceptación & $\begin{array}{c}\text { Autonomía } \\
\text { extrema }\end{array}$ \\
\hline Reestructuración cognitiva & -.017 & -.013 & -0.207 \\
Autoinculparse & $\mathbf{- 0 . 6 2 0 * *}^{\text {** }}$ & $\mathbf{0 . 5 6 6}^{* *}$ & 0.170 \\
Fatalismo & -0.286 & 0.146 & 0.149 \\
Evasión a través de la diversión & -0.201 & 0.146 & .085 \\
Focalizado en el problema & -0.202 & 0.251 & -.072 \\
Evasión a través de la actividad física & .068 & -0.134 & .025 \\
Apoyo emocional & -0.326 & 0.323 & -.027 \\
Descarga emocional y somatización & -0.221 & 0.261 & 0.163 \\
Ansiedad & -0.325 & 0.254 & -0.133 \\
Aislamiento & $\mathbf{- 0 . 3 9 8}$ & 0.167 & 0.113 \\
No acción & -0.300 & .030 & 0.161 \\
\hline
\end{tabular}

$* \mathrm{p}<.05 ; * \mathrm{p}<.01$

\section{Análisis complementario.}

Se realizaron análisis de comparación de medias de las variables de estudio para determinar la existencia de diferencias de acuerdo con el sexo. Al analizar las estrategias de afrontamiento en relación con el sexo (Tabla 4), no se encontraron algunas diferencias estadísticamente significativas. 
Tabla 4.

Diferencias en el estilo de afrontamiento según sexo de los hijos.

\begin{tabular}{lccccccc}
\hline \multirow{2}{*}{\multicolumn{1}{c}{ Escala }} & \multicolumn{2}{c}{$\begin{array}{c}\text { Femenino } \\
(\mathbf{n = 1 6 4 )}\end{array}$} & \multicolumn{2}{c}{$\begin{array}{c}\text { Masculino } \\
(\mathbf{n}=\mathbf{1 5 6})\end{array}$} & \multirow{2}{*}{ t } & Sig. \\
\cline { 2 - 6 } & $\mathbf{M}$ & $\mathbf{D . E}$ & $\mathbf{M}$ & $\mathbf{D . E .}$ & & \\
\hline Reestructuración cognitiva & 3.56 & 0.82 & 3.81 & 0.79 & 0.752 & 0.459 \\
Autoinculparse & 2.09 & 0.74 & 2.08 & 0.96 & -.034 & 0.973 \\
Fatalismo & 2.37 & 0.72 & 2.16 & 1.16 & -0.600 & 0.554 \\
Evasión a través de la diversión & 3.27 & 0.79 & 3.50 & 0.82 & 0.676 & 0.505 \\
Focalizado en el problema & 3.44 & 0.63 & 3.48 & 0.72 & 0.134 & 0.895 \\
Evasión a través de la actividad física & 2.65 & 0.89 & 3.16 & 0.94 & 1.339 & 0.192 \\
Apoyo emocional & 3.33 & 0.94 & 3.19 & 0.85 & -0.381 & 0.706 \\
Descarga emocional y somatización & 2.49 & 0.67 & 2.38 & 1.00 & -0.354 & 0.726 \\
Ansiedad & 3.46 & 0.64 & 3.25 & 0.80 & -0.749 & 0.460 \\
Aislamiento & 2.39 & 0.66 & 2.97 & 0.73 & 2.037 & .052 \\
No acción & 1.89 & 0.68 & 2.06 & 0.88 & 0.592 & 0.559 \\
\hline
\end{tabular}

La Tabla 5 presenta las diferencias encontradas en la percepción de la relación con el padre de acuerdo con el sexo. Se observa que solamente existen diferencias significativas en autonomía extrema. Esta diferencia fue baja $(d=0.25)$, en la cual los varones puntúan más alto que las mujeres.

\section{Tabla 5.}

Diferencias en la percepción de la relación con el padre según el sexo de los hijos.

\begin{tabular}{lccccccc}
\hline \multirow{2}{*}{ Escala } & \multicolumn{2}{c}{$\begin{array}{c}\text { Femenino } \\
(\mathbf{n = 1 6 4})\end{array}$} & \multicolumn{2}{c}{$\begin{array}{c}\text { Masculino } \\
(\mathbf{n}=\mathbf{1 5 6})\end{array}$} & \multirow{2}{*}{ t } & Sig. \\
\cline { 2 - 6 } & $\mathbf{M}$ & $\mathbf{D . E .}$ & $\mathbf{M}$ & $\mathbf{D . E}$ & & \\
\hline Control patológico & 18.57 & 5.05 & 18.5 & 2.88 & -.038 & 0.97 \\
Aceptación & 23.95 & 7.5 & 23 & 6.59 & -0.315 & 0.755 \\
Autonomía extrema & 17.14 & 3.61 & 20.63 & 4.03 & 2.25 & $.03^{*}$ \\
\hline
\end{tabular}

$* \mathrm{p}<.05$ 
Respecto a la percepción de la relación con la madre (Tabla 6), podemos observar que no se evidencian diferencias estadísticamente significativas entre hombres y mujeres.

\section{Tabla 6.}

Diferencias en la percepción de la relación con la madre según el sexo de los hijos.

\begin{tabular}{|c|c|c|c|c|c|c|}
\hline \multirow[t]{2}{*}{ Escala } & \multicolumn{2}{|c|}{$\begin{array}{c}\text { Femenino } \\
(n=164)\end{array}$} & \multicolumn{2}{|c|}{$\begin{array}{c}\text { Masculino } \\
(n=156)\end{array}$} & \multirow[t]{2}{*}{$\mathbf{t}$} & \multirow[t]{2}{*}{ Sig. } \\
\hline & $\mathbf{M}$ & D.E. & $\mathbf{M}$ & D.E. & & \\
\hline Control patológico & 22.43 & 2.98 & 18.38 & 5.78 & -1.890 & .093 \\
\hline Aceptación & 23.81 & 7.06 & 28.13 & 6.15 & 1.51 & 0.141 \\
\hline Autonomía extrema & 17.57 & 3.12 & 18.25 & 1.83 & .574 & 0.571 \\
\hline
\end{tabular}

\section{Discusión}

La presente investigación examina la correlación entre la percepción de la relación con los padres y los estilos de afrontamiento en un grupo de adolescentes universitarios de Lima. Los resultados reportan que algunos estilos de las relaciones con los padres están vinculados con las estrategias de afrontamiento por parte de los adolescentes.

Respecto a las relaciones entre la aceptación del padre y de la madre con las estrategias de afrontamiento, encontramos que un mayor empleo de estrategias caracterizadas porque la persona se siente responsable de los problemas que le aquejan se asocia con una menor percepción de control hostil y con una mayor aceptación por parte de la madre. Nuestros resultados concuerdan con Richaud de Minzi, Moreno y Sacchi (2011), quienes mencionan que una percepción de aceptación por parte de los padres es un factor protector para el desarrollo de afrontamientos basados en la evitación cognitiva. La relación entre la aceptación de la madre y las estrategias centradas en la emoción es importante pues se vinculan con bajos niveles 
de rendimiento, así como con el desarrollo de síntomas psicosomáticos y sentimientos de culpa y autocrítica, si es que no puede hacer frente al problema (Canessa, 2002).

En casos como los observados en relación con la madre, en donde se percibe una aceptación de su parte junto con bajos niveles de control patológico, la responsabilidad por los problemas aquejados se encuentra acompañada de cuadros de ansiedad que, a su vez, aumentan la probabilidad de emplear estrategias de búsqueda de apoyo (Marcoen, Goossens \& Caes, 1987), asociación ausente en nuestra muestra.

Con respecto a las hipótesis referidas a la posible relación entre el control patológico del padre y de la madre y las estrategias de afrontamiento, observamos que, en el caso del padre, un mayor empleo de estrategias caracterizadas por un cambio de actitud positiva se asocia a una menor percepción de control hostil por parte de los padres. De acuerdo con Richaud de Minzi (2006a, 2006b), la reestructuración cognitiva actúa sobre el problema real y la búsqueda de apoyo emocional. Este resultado estaría relacionado con lo expuesto por Richaud de Minzi, Moreno y Sacchi (2011), para quienes la disminución de un control hostil, junto con una mayor aceptación, permitiría desarrollar un afrontamiento basado en la reestructuración cognitiva, la búsqueda de apoyo emocional y la acción sobre el problema.

Así mismo, la ausencia de control excesivo por parte de ambos padres estaría relacionada con el mayor empleo de estilos de afrontamiento centrado en la evaluación que sugieren que, cuando no se puede modificar la situación problemática, es más factible hacerla cognitivamente más manejable (Richaud de Minzi, 2005). Lo anterior se halla en contraposición a lo señalado por Wolfradt, Hempel y Miles (2003), para quienes aquellas personas que percibían la relación con sus padres como autoritaria muestran mayores puntuaciones en el afrontamiento activo a los problemas. 
En nuestros resultados se observa también una correlación negativa entre el control patológico y actividades de aislamiento, lo cual sugiere que una mayor percepción de control hostil por parte de los padres se asocia con una disminución de actividades de escape, huida y falta de responsabilidad del problema asociado a la soledad (Richaud de Minzi, 2006a). Esta soledad no facilitaría la reflexión y el sentimiento de tranquilidad, ya que estaría asociada a sentimientos de desconfianza y rechazo hacia los demás (Richaud de Minzi, 2002). Otros autores (Richaud de Minzi, 2002) señalan que el control excesivo o patológico puede conducir a los hijos a sentirse rechazados, afectando su acercamiento a los pares. Nuestros resultados estarían acorde con Alíes- Jardel, Fourdrinier, Roux y Schneider (2002), quienes afirman que ciertas restricciones son necesarias pues permiten el establecimiento de adecuadas relaciones entre pares. Así, una percepción de los padres de mayor firmeza y vigilancia estaría asociada con el empleo de afrontamientos centrados en la emoción (Richaud de Minzi, 2002); mientras que un control insuficiente, junto con una autonomía extrema por parte de los padres, podría estar asociado con la presencia de conductas antisociales (Dishion, 1990).

Finalmente, en relación con la posible asociación entre la percepción de la autonomía extrema del padre y de la madre con las estrategias y estilos de afrontamiento, encontramos solo una relación significativa en el caso del padre con la estrategia denominada ansiedad, lo que indica que un mayor empleo de acciones que expresan un temor por el futuro se asocia con un menor control por parte de los padres. De acuerdo con Wolfradta, Hempelb $\&$ Miles (2003), la ansidedad estaría a su vez relacionada de manera negativa con la percepción de calidez parental. De la misma forma, Richaud de Minzi, Moreno y Sacchi (2011) señalan que, si bien el control es importante, un estilo parental caracterizado por el control excesivo puede llegar a limitar la autonomía y la seguridad del adolescente, siendo nada beneficioso para llevar a cabo un afrontamiento exitoso del problema. Los hijos que perciben una carencia de protección, debido a una falta de control y de límites, experimentan mayores niveles de descontrol emocional (Richaud de Minzi et al., 2013). 
Respecto a las diferencias del afrontamiento en relación con el sexo, no se hallaron diferencias estadísticamente significativas, lo que no guarda relación con la literatura peruana al respecto (Canessa, 2000; Cassaretto, Chau, Oblitas \& Valdés, 2003; Casuso, 1996; Martínez \& Morote, 2001; Cassaretto, 2009), para quienes las mujeres emplean con mayor frecuencia el apoyo social, acudiendo continuamente a la religión; mientras que los varones suelen utilizar, en mayor medida, estrategias activas en comparación con las mujeres.

Finalmente, en relación con las comparaciones de la percepción de los estilos parentales respecto al sexo, podemos observar que, en el caso del padre, existen diferencias significativas en autonomía extrema, en la cual los varones puntúan más alto que las mujeres; es decir, los hijos perciben una mayor falta de control por parte de los padres. Este resultado coincide con diferentes estudios que señalan que los varones perciben a sus padres más negligentes en comparación con las mujeres (Hines, Kantor \& Holt, 2006; Shek, 1998, 2000). Otro estudio (Rodriguez, Del Barrio \& Carrasco, 2009) indica también que los varones perciben a sus padres como más controladores, permisivos y hostiles. Lo anterior nos lleva a considerar que los varones son más sensibles a la percepción del control y la disciplina (Fuentes, Motrico \& Bersabé, 2001; Mestre, Samper, Tur \& Díez, 2001).

Los resultados en relación con el sexo y la percepción de los estilos parentales llevan a sostener el estereotipo de "padre estricto y madre cariñosa" (Shek, 1998) pues, si bien se percibe a las madres como más afectivas y a los padres como exigentes, los resultados no son estadísticamente significativos, tal como informan otros autores (Shek, 2000; Sorbring, Rödholm-Funnemark \& Palmerus, 2003). A pesar de esto, la literatura científica referida a la percepción de la relación con los padres respecto al sexo no se encuentra libre de controversia, tal como lo demuestran numerosos resultados (Forehand \& Nousiaien, 1993; Musitu \& Cava, 2001; Shek, 1998). Esta controversia puede ser causada por los cambios acontecidos en el estilo educativo de padres y madres (Lanz, Scabini, Vermulst \& Gerris, 2001; Sorbring et al., 2003). 
Los resultados del estudio sugieren la relación entre los patrones de interacción entre los padres y los niños y las estrategias de afrontamiento, variables importantes en la adaptación de los adolescentes. Sin embargo, es necesario estudiar cómo el hecho de que predomine uno u otro estilo depende del ajuste entre las condiciones psicosociales en las que vive la familia, el escenario educativo que los padres o cuidadores han construido y las características de los mismos.

Los resultados de esta investigación deben ser considerados a la luz de las siguientes limitaciones. En primer lugar, en este trabajo solo se consideró la participación de los constructos percepción de la relación con los padres, dejando de lado otros constructos familiares importantes al momento de emplear determinadas estrategias de afrontamiento. En este sentido, sería importante desarrollar en el futuro nuevos estudios que evalúen de forma conjunta la contribución de diversas variables familiares en relación con el afrontamiento de los adolescentes y jóvenes en el contexto peruano. Segundo, al ser la muestra extraída de una población universitaria limeña mediante un muestreo por conveniencia, los resultados tienen limitada capacidad de generalización al resto del país dadas las diferencias culturales y el estilo de vida presentes en costa, sierra y selva. Tercero, es necesario contar con una mayor cantidad de participantes y de diferentes sectores sociales en próximas investigaciones, con el fin de poner a prueba el modelo de regresión. 


\section{Referencias}

Allès-Jardel, M., Fourdrinier, C., Roux, A., \& Schneider, B. H. (2002). Parents' structuring of children's daily lives in relation to the quality and stability of children's friendships. International Journal of Psychology, 37, 65-73. doi: 10.1080/00207590143000289

Baumrind, D. (1966). The discipline controversy revisited. Family Relations, 45(4), 405-414. doi: 10.2307/585170

Broderick, P. C., \& Blewitt, P. (2003). The life span: Human development for helping professionals. Upper Saddle River, NJ: Pearson Education.

Caballero, C., Abello, R., \& Palacios, J. (2007). Relación del burnout y el rendimiento académico con la satisfacción frente a los estudios en estudiantes universitarios. Avances en Psicología Latinoamericana, 25(2), 98-111.

Calais, S. L., Andrade, L. M. B., \& Lipp, M. E. N. (2003). Diferenças de sexo e escolaridade na manifestaçao de stress em adultos jovens. Psicología: Reflexâo e Crítica, 16(2), 257-263.

Canessa, B. (2002). Adaptación psicométrica de las Escalas de Afrontamiento para adolescentes de Frydenberg y Lewis en un grupo de escolares de Lima Metropolitana. Persona, 5, 191-233.

Cassaretto, M., Chau, C., Oblitas, H., \& Valdés, N. (2003). Estrés y afrontamiento en estudiantes de psicología. Revista de Psicología, 21(2), 363-392.

Cassareto, M. (2009). Relación entre las cinco grandes dimensiones de la personalidad y el afrontamiento en estudiantes preuniversitarios de Lima Metropolitana (Tesis de maestría). Universidad Nacional Mayor de San Marcos, Lima.

Casuso, L. (1996). Adaptación de la prueba COPE sobre estilos de afrontamiento en un grupo de estudiantes universitarios de Lima (Tesis de licenciatura inédita). Pontificia Universidad Católica del Perú, Lima.

Caycho, T., Castilla, H., \& Shimabukuro, M. (2014). Afrontamiento en adolescentes peruanos: análisis confirmatorio de la Adolescent Coping Scale, versión abreviada. Eureka, 11(2), 187-203. 
Cohen, S., Kessler, R., \& Underwood, L. (1995). Strategies for measuring stress in studies of psychiatric and physical disorders. En S. Cohen, R. Kessler y L. Underwood (Eds.), Measuring Stress: A Guide for Health and Social Scientists (pp. 3-20). New York: Oxford.

Darling, N., \& Steinberg, L. (1993). Parenting style as context: An integrative model. Psychological Bulletin, 113 (3), 487-496. doi: 10.1037/00332909.113.3.487

Dishion, T. J. (1990). The family ecology of boys peer relations in middle school. Child Development, 6(3), 874-892. doi: 10.1111/j.14678624.1990.tb02829.x

Domenech, M., Donovick, R., \& Crowley, S. (2009). Parenting styles in a cultural context: Observations of "protective parenting" in firstgeneration latinos. Family Process, 48(2), 1-18. doi: 10.1111/j.15455300.2009.01277.x

Dusek, J., \& Danko, M. (1994). Adolescent Coping Styles and Perceptions of Parental Child rearing, Journal of Adolescent Research, 9(4), 412426. doi: $10.1177 / 074355489494002$

Forehand, R., \& Nousiainen, S. (1993). Maternal and paternal parenting: Critical dimensions in adolescent functioning. Journal of Family Psychology, 7(2), 213-221. doi: 10.1037/0893-3200.7.2.213

Frydenberg, E. (1993). The coping strategies used by capable adolescents. Australian Journal of Guidance \& Couselling, 3(1), 1-9.

Frydenberg, E. (1997). Adolescent Coping. New York: Routledge.

Frydenberg, E., \& Lewis, R. (1997). ACS Escalas de Afrontamiento para adolescentes. Adaptación española de Jaime Pereña y Nicolás Seisdedos. [ACS Adolescent Coping Scales. Spanish version of Jaime Pereña Seisdedos]. Madrid: TEA Ediciones.

Fuentes, M. J., Motrico, E., \& Bersabé, R. M. (2001). Diferencias entre padres y adolescentes en la percepción del estilo educativo parental: afecto y normas-exigencias. Apuntes de Psicología, 19, 235-249.

Guarino, L., Gavidia, I., Antor, M. \& Caballero, H. (2000). Estrés, salud mental y cambios inmunológicos en estudiantes universitarios. Psicología Conductual, 8, 57-71. 
Heiman, T. (2004). Examination of the Salutogenic Model, Support Resources, Coping Style, and Stressors Among Israeli University Students. The Journal of Psychology, 138(6), 505-520. doi: 10.3200/ JRLP.138.6.505-520

Herman, M. A., \& McHale, S. M. (1993). Coping with parental negativity: Links with parental warmth and child adjustment. Journal of Applied Development Psychology, 14(1), 131-136. doi: 10.1016/01933973(93)90027-S

Hernández, R., Fernández, C., \& Baptista, L. (2006). Metodología de la Investigación. México: McGraw-Hill/Interamericana.

Hines, D., Kantor, G., \& Holt, M. (2006). Similarities in siblings' experiences of neglectful parenting behaviours. Child Abuse and Neglect, 30(6), 619637. doi: 10.1016/j.chiabu.2005.11.008

Lanz, M., Scabini, E., Vermulst, A. A., \& Gerris, J. R. (2001). Congruence on child rearing in families with early adolescent and middle adolescent children. International Journal of Behavioral Development, 25(2), 133139. doi: $10.1080 / 01650250042000104$

Lazarus, R., \& Folkman, S. (1986). Estrés y procesos cognitivos. Barcelona: Martínez Roca.

Maccoby, E., \& Martin, J. (1983). Socialization in the context of the family: Parent-child interaction. En P. H. Mussen (Series Ed.) \& E. M. Hetherington (Vol. Ed.), Handbook of child psychology, 4. Socialization, personality, and social development (4th ed., pp. 1-101). New York: Wiley.

Marcoen, A., Goossens, L., \& Caes, P. (1987). Loneliness in pre-through late adolescence: exploring the contributions of a multidimensional approach. Journal of Youth and Adolescence, 16(6), 561-577. doi: 10.1007/BF02138821

Martín, I. M. (2007). Estrés académico en estudiantes universitarios. Apuntes de Psicología, 25(1), 87-99.

Martínez, P., \& Morote, R. (2001). Preocupaciones de adolescentes de Lima y sus estilos de afrontamiento. Revista de Psicología, 19(2), 211-236. 
Mestre, M. V., Samper, P., Tur, A., \& Díez, I. (2001). Estilos de crianza y desarrollo pro social de los hijos. Revista de Psicología General y Aplicada, 54, 691-703.

Musitu, G., \& Cava, M. J. (2001). El rol del apoyo social en el ajuste de los adolescentes. Intervención Psicosocial, 12, 179-192.

Richaud de Minzi, M. C. (2002, julio). Loneliness and depression in children: its relationship with attachment and self competence. En M.e. Richaud de Minzi (presidente), Stress and emotions in children and adolescents. Invited Symposium efectuado en la reunión del XXV Intemational Congress of Applied Psychology, Singapur.

Richaud de Minzi, M. C. (2003). Coping Assessment in adolescents. Adolescence, 38(150), 321-330.

Richaud de Minzi, M. C. (2005). Versión abreviada del Inventario de la Percepción de los Hijos Acerca de las Relaciones con sus Padres para Adolescentes. Psicodiagnosticar, 15, 99-106.

Richaud de Minzi, M. C. (2006a). Stress and coping in adolescence. In A. M. Columbus (Ed.), Advances in Psychology Research, Volume 45 (pp. 67-84). Hauppauge, NY: NovaEditorial Publishers.

Richaud de Minzi, M. C. (2006b). Evaluación del afrontamiento en niños de 8 a 12 años. Revista Mexicana de Psicología, 23(2), 196-201.

Richaud de Minzi, M. C., Moreno, J. E., \& Sacchi, C. (2011). Un modelo acerca de la relación entre los vínculos interpersonales y el afrontamiento en la adolescencia. En M. C. Richaud y V. Lemos (Eds.), Psicología y otras Ciencias del Comportamiento. Compendio de investigaciones actuales Vol. 1 (pp. 137-157). Ediciones CIIPME-CONICETy Universidad Adventista del Plata Ediciones.

Richaud de Minzi, M. C., Mesurado, B., Samper-García, P., Llorca, A., Lemos, V., \& Tur, A. (2013). Estilos parentales, inestabilidad emocional y agresividad en niños de nivel socioeconómico bajo en Argentina y España. Ansiedad y Estrés, 19(1), 53-69.

Rodríguez, M. A., Del Barrio, M. V., \& Carrasco, M. A. (2009). ¿Cómo perciben los hijos la crianza materna y paterna? Diferencias por edad y sexo. Escritos de Psicología, 2(2), 10-18. 
Sandler, I. N., Wolchick, S. A., MacKinnon, D., Ayers, T. S., \& Roosa, M. W. (1997). Developing linkages between Theory and Intervention in Stress and Coping Processes. En S. A. Wolchick \& I. N. Sandler (Eds.), Handbook of Children's Coping. LinkingTheory and Intervention (pp. 3-40). Nueva York: Plenum Press.

Sarid, O., Anson, O., Yaari, A., \& Margalith, M. (2004). Academic Stress, Immunological Reaction and Academic Performance among Students of Nursing and Research. Nursery Health, 27(5), 370-377. doi: 10.1002/ nur. 20028

Schaefer, E. S. (1965). Children's reports of parental behavior: An Inventory, Child Development, 38(2), 413-424. doi: 10.2307/1126465

Shek, D. (1998). Perceptions of parenting styles and parent-adolescent conflict in adolescents with low academic achievement in Hong Kong. Social Behavior and Personality, 26(1), 89-98. doi: 10.2224/sbp.1998.26.1.89

Shek, D. (2000). Differences between fathers and mothers in the treatment of, and relationship with, their teenage children: perceptions of Chinese adolescents. Adolescence, 35, 135-146.

Skinner, E. A., \& Welborn, J. G. (1994). Coping during childhood and adolescence: A motivational perspective. En R. Lerner, D. Featherman \& M. Perlmutter (eds.), Lifespan development and behavior (Vol. 12, pp. 91-123). Hillsdale, NJ: Lawrence Earlbaum Associates.

Sorbring, E., Rödholm-Funnemark, M., \& Palmerus, K. (2003) Boys' and gilrs' perceptions of parental discipline in transgression situations. Infant and Child Development, 12(1), 53-69. doi: 10.1002/icd.265

Taylor, S. (2007). Psicología de la Salud. México: McGraw-Hill/ Interamericana.

Wolfradt, U., Hempelb, S., \& Miles, J. N. V. (2003). Perceived parenting styles, depersonalisation, anxiety and coping behaviour in adolescents. Personality and Individual Differences, 34(3), 521-532. doi: 10.1016/ S0191-8869(02)00092-2 